PHYSICAL REVIEW E 84, 037302 (2011)

\title{
Azimuthal solitary surface wave in cylindrical tank
}

\author{
Hamid Ait Abderrahmane* \\ Department of Mechanical Engineering, McGill University, Montréal, Québec, Canada H3A 2K6 \\ Mustapha Amaouche \\ Laboratoire de Physique Nonlineaire, Université de Béjaia, Algeria
}

Mohamed Fayed, Hoi Dick Ng, and Georgios H. Vatistas

Department of Mechanical and Industrial Engineering, Concordia University, Montréal, Québec, Canada H3G 1 M8

Kamran Siddiqui

Department of Mechanical and Materials Engineering, University of Western Ontario, London, Ontario, Canada N6A 5B9

(Received 5 July 2011; revised manuscript received 12 August 2011; published 22 September 2011)

\begin{abstract}
This Brief Report is devoted to the study of the solitary surface wave rotating in the azimuthal direction, arising during water drainage from a cylindrical reservoir, when shallow flow conditions are reached. The linear dependence between the wave speed and its amplitude is shown to be similar to that expected from the classical Korteweg-de Vries equation.
\end{abstract}

DOI: 10.1103/PhysRevE.84.037302

PACS number(s): 47.35.Fg

Following its discovery in 1844 by Russell [1], the solitary wave has been the subject of intense research because of its manifestations in several fields of physics such as acoustic waves, magnetoacoustics, and several others; see [2-4]. Solitary waves were also observed in rotating flow and it is known that the background rotation significantly influences the propagation of both surface and internal gravity solitary waves. It is also found to be responsible for the creation of the inertial waves. This dual impact of rotation can be illustrated through two relevant physical cases.

The first concerns internal gravity waves developed in a shallow rotating channel where the influence of rotation depends on its strength; see [5]. In the case of intense rotation, the influence is distinguishable from those of weak nonlinearity and dispersion; here the result is a transverse exponential decay of the solitary wave amplitude. However, when the rotation level becomes of the same order of magnitude as the nonlinearity and dispersion, the relevant governing equation is the rotation-modified Kadomstev-Petriashvili (KP) instead of the classical Korteweg-de Vries (KdV); see [5-7]. At the limit of infinitely large channel width, the KP equation reduces to the Ostrovsky equation, which was first derived to account for the effects of the earth's rotation on oceanic internal solitary waves [8].

The second relates to the situation where rotation induces inertial waves as in the case of a rotating homogeneous fluid in a rigid long tube [9]. The last flow configuration indicates that solitary wave solutions of the $\mathrm{KdV}$ equation are possible in any swirling flow in which the angular velocity is nonuniformly distributed $[5,9,10]$. Inertial solitary waves have also been observed within the swirling flow produced in a large vertical cylindrical container. This wave is seen to propagate up and down between the bottom of the container and the free liquid surface, along the vortex core [9].

\footnotetext{
*haitabd@hotmail.com
}

In this communication we report on yet another type of solitary wave within rotating flow. This wave is observed during the liquid drainage through a centrally located circular opening on the bottom of the container, when shallow water conditions are reached. The senior author initially reported the existence of this wave (only parenthetically) twenty-one years ago [11]. This wave manifestation is examined systematically via both theory and experiment.

The experiments were performed in a Plexiglas cylindrical container of diameter $D$ equal to $285 \mathrm{~mm}$. The drainage hole of diameter $d$ equal to $31.7 \mathrm{~mm}$ was located at the bottom center of the container. The tank was filled with water and two initial water heights, $H i=435$ and $H i=270 \mathrm{~mm}$, were considered. A blue water soluble food color was added to the fluid prior to the experiments for better visualization. A schematic of the experimental setup is shown in Fig. 1. Water within the tank was stirred with a rod, in a way similar to that of [9], at known rotations per minute (i.e., $\omega=33,60$, $70,92,100$, and $120 \mathrm{rpm}$ ), producing different initial swirls. Soon after, the rod was lifted and the bottom manifold was opened for water drainage. As the water level decreased, the amplitude of the oscillations of the free surface increased; these oscillations matured into a single surface bulge (solitary wave) when the shallow-water conditions were reached. At this time, we replugged the hole; therefore the water depth remained once again constant, and the solitary wave revolved around the cylindrical wall till it vanished. It is worth noting that a solitary wave could be observed only when either an initial swirl was imparted to the liquid or when a residual vorticity (to reservoir filling-up process) was present in the initial stages of the draining process. In fact, if the tank is filled and then left undisturbed for a while to calm down prior to draining (i.e., almost no residual vorticity), the solitary wave may not appear at all. The event was recorded using a CCD camera; the images were acquired at a rate of $90 \mathrm{~Hz}$, respectively. The camera resolution was $1600 \times 1200$ and the shutter speed set equal to $1 / 500$ seconds to avoid blurring effects. The image acquisition 


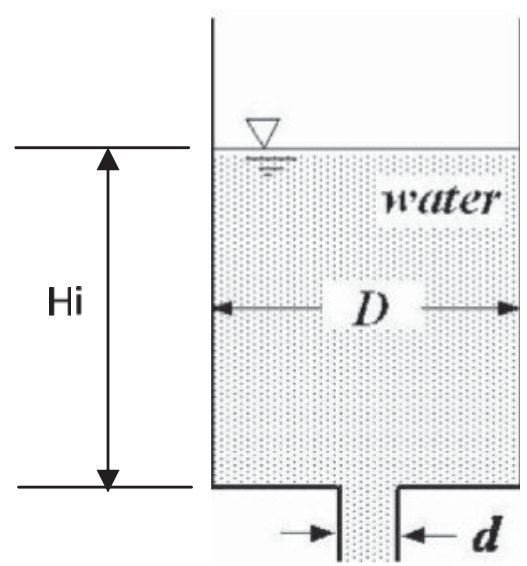

Camera

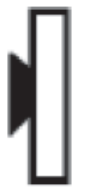

FIG. 1. Experimental setup. Plexiglas cylindrical reservoir with a central drainage hole located at the bottom center of the container.

started immediately after the opening of the manifold and continued until the drainage stopped, just after the solitary wave arose. Images at the last stages of the drainage process and the formation of the revolving solitary wave are illustrated in Fig. 2 (and the Supplementary Material [12]).

The free surface first displayed long wavelength undulations as seen in Fig. 2(a). The amplitude of the wave increased as the water height decreased. When the shallow water conditions were reached (water height smaller that the container diameter), the free surface undulations transformed almost instantly into a surface bulge or solitary wave rotating around the cylindrical wall of the container [see Figs. 2(c)-2(f)]. During all experimental runs, the solitary wave was formed when the water height reached between $H_{0}=25 \mathrm{~mm}$ and $H_{0}=40 \mathrm{~mm}$ which correspond to a shallow water parameter $\left(H_{0} / D\right)$ varying between 0.088 and 0.157 , respectively.

To accurately detect the wave profile, digital image processing was performed. The temporal variation of the free surface profile was obtained by measuring the free surface elevation in reference to the bottom of the container at a fixed angular location on the free surface with respect to the tank cross section. Due to the axisymmetric geometry, there is no preference for the selection of this location. For convenience in the analysis, the angular location that corresponds to the middle section of the images was selected.

The classical $\mathrm{KdV}$ equation arises from the governing equations of irrotational motion of an incompressible inviscid fluid bounded above by a free surface and below by a rigid

(a)

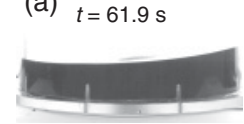

(b) $t=64.7 \mathrm{~s}$
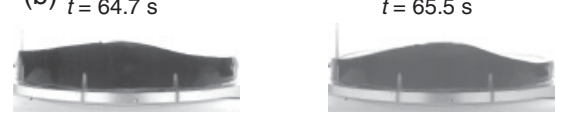

FIG. 2. Example of a maturation of the free surface oscillation into bulge (solitary wave). Initial water height $H i=435 \mathrm{~mm}$; initial swirl $\omega=70 \mathrm{rpm}$. Time $t=0$ corresponds to the beginning of the drainage. horizontal plane. In the case under consideration the wave motion emerges from a primary rotating flow in which a swirl velocity is imparted which yields a certain radial distribution of the fluid layer thickness. The resulting perturbed motion is rotational and accordingly must be described by the Euler equations. Similarly to the basic flow, the perturbed velocity is assumed to have no radial component. Then the governing dimensionless equations read

$$
\begin{gathered}
\frac{1}{r} v_{\theta}+w_{z}=0, \\
\epsilon\left\{D_{t} v+\epsilon\left(\frac{1}{r} v v_{\theta}+w v_{z}\right)\right\}+\frac{1}{r} p_{\theta}=0, \\
\epsilon \delta\left\{D_{t} w+\epsilon\left(\frac{1}{r} v w_{\theta}+w w_{z}\right)\right\}+p_{z}+1=0 .
\end{gathered}
$$

These equations are subject to the boundary conditions

$$
\left.p\right|_{z=h_{0}+\epsilon \eta}=0,\left.\quad w\right|_{z=0}=0,\left.\quad w\right|_{z=h_{0}+\epsilon \eta}=D_{t} \eta+\epsilon \frac{1}{r} v \eta_{\theta},
$$

and are written in cylindrical coordinates $(r, \theta, z) ; v$ and $w$ are the azimuthal and axial velocity components, respectively, $p$ is the pressure, and $\eta$ indicates the free surface elevation. The parameters appearing in Eqs. (1)-(4) are $\epsilon=\frac{A}{H_{0}}$ and $\delta=\left(\frac{H_{0}}{R}\right)^{2}$ where $A$ is a measure of the wave amplitude, $R$ is the cylinder radius which serves to scale horizontal distances, and $H_{0}$ (the typical length scale in the axial direction) is the undisturbed fluid depth at the wall. Velocity components $v$ and $w$ and time and pressure are in units of $\epsilon \sqrt{g H_{0}}, \epsilon \sqrt{g H_{0} / \delta}$, $R / \sqrt{g H_{0}}$, and $\rho g H_{0}$, respectively. The symbol $D_{t}$ stands for the total time derivative $\partial_{t}+\Omega \partial_{\theta}$ where $\Omega$ denotes the relative background rotation in comparison with $\sqrt{g H_{0}} / R$, and $h_{0}=1+\frac{1}{2} \Omega^{2}\left(r^{2}-1\right)$ is the local scaled depth. We are interested in small amplitude waves, i.e., in the limits as $\epsilon \rightarrow 0$ and $\delta \rightarrow 0$. Similar to the planar case, it turns out that one choice which leads to the $\mathrm{KdV}$ equation for $\eta$ is to set $\delta=O(\epsilon)$ as $\epsilon \rightarrow 0$. Since the radial coordinate only appears as a parameter in the governing equation, the $\mathrm{KdV}$ equation can be derived following the classical approach; this leads to

$\eta_{t}+\left(\sqrt{h_{0}}+\Omega r+\frac{3}{2} \frac{\epsilon}{\sqrt{h_{0}}} \eta\right) \eta_{x}+\frac{\delta}{6} h_{0}^{2} \sqrt{h_{0}} \eta_{x x x}=0$,

where the curvilinear coordinate $x=r \theta$ is used instead of $\theta$. It has, in dimensional form, the solution

$$
\begin{aligned}
\eta(r, x, t)= & a(r) \operatorname{sech}^{2} \sqrt{\frac{3}{4} \frac{a(r)}{h_{0}^{3}}} \\
& \times\left\{x-\left(\sqrt{g h_{0}}+\Omega r+\frac{1}{2} \frac{a(r) \sqrt{g h_{0}}}{h_{0}}\right) t\right\} .
\end{aligned}
$$

If the radial distribution of the wave amplitude, $a(r)$, is such that the phase celerity does not depend on $r$, i.e., $\left(\sqrt{g h_{0}}+\right.$ $\left.\Omega r+\frac{1}{2} \frac{a(r) \sqrt{g h_{0}}}{h_{0}}\right)=$ const, the above solution represents a 

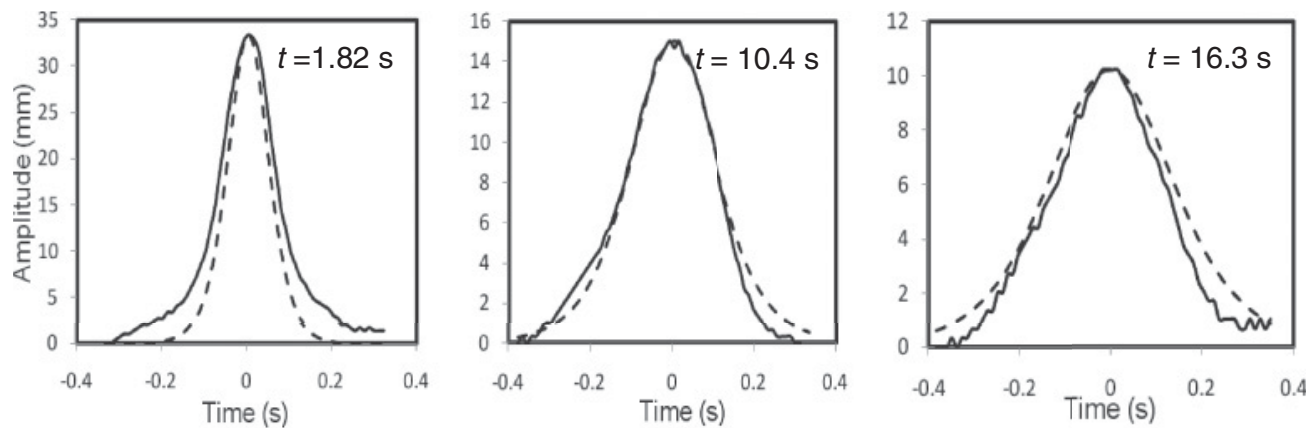

FIG. 3. Snapshots of free surface elevation at three different times and at a constant water depth $H_{0}=25.5 \mathrm{~mm}$. Initial swirl $\omega=120 \mathrm{rpm}$ and initial water height $\mathrm{Hi}=435 \mathrm{~mm}$. Time $t=0$ corresponds to the formation of the bulge. (Solid line: experiment; dashed line: theory.)

traveling solitary wave; the constant can be determined using the wave characteristics at the wall $\left(h_{0}=H_{0}, a=A\right)$. Otherwise, the solution (6) might be interpreted as a juxtaposition of a series of traveling solitary waves at each radial station, each of them with its own characteristics. A more elaborated analysis of the radial wave dependence requires the consideration of the influence of the radial flow. Here our interest is only with the wall wave motion which can be done by the projection of the equation (5) on the wall cylinder.

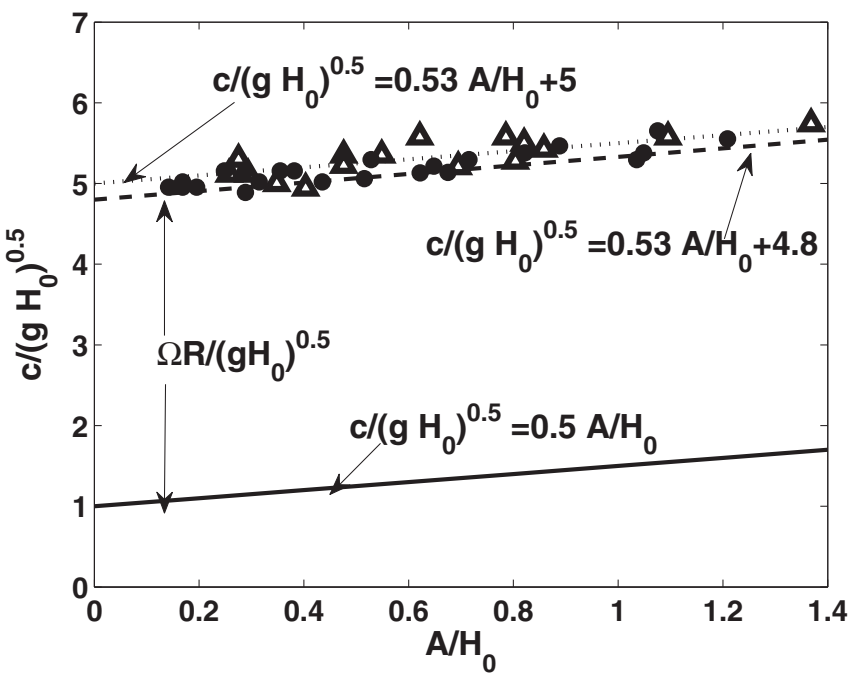

FIG. 4. Amplitude-dependent wave speed for constant water depth case. Experimental conditions as follows. Run 1 (dots): $H_{0}=25.5 \mathrm{~mm}$ with initial swirl $\omega=120 \mathrm{rpm}$ and initial water height $H i=435 \mathrm{~mm}$. Run 2 (triangles): $H_{0}=18 \mathrm{~mm}$ with initial swirl $\omega=90 \mathrm{rpm}$ and initial water height $H i=435 \mathrm{~mm}$. The dashed lines are the linear best fits of the experimental data.
In Fig. 3 we compare the experimental and theoretical solitary wave profiles at three different instances. The experimental wave profiles are symmetrical at all times and agree fairly well with the theoretical profiles. Note that $\Omega$ is an unknown background flow angular velocity; its value was determined in such a way as to harmonize the experiment with the theory. The slight discrepancies between the experimental and the theoretical profiles in particular at the beginning and the end of the wave's lifetime can be attributed to the fact that water is a viscous fluid while the theory considers inviscid flow. Moreover, the friction at the cylindrical wall should affect also the wave profile. From the results shown in Fig. 3, one can ascertain that the observed wall wave coincides with a $\mathrm{KdV}$ solitary wave profile.

The wave speed was calculated using subsequent snapshots of the wave when it passes in front of the cameras. Measuring the distance between the waves' crests and knowing the time between two snapshots, the wave speed can be deduced. The dimensionless wave speed is displayed as a function of $A / H_{0}$ for various initial heights and initial swirl velocities in Fig. 4. The results show that the dimensionless wave speed varies linearly and the slope of the linear best fit of the experimental data is 0.53 , which is very close to the theoretical slope of 0.5 as determined by the theory.

In conclusion this communication reported a study of a revolving solitary wave, observed during the draining of a water-filled tank when shallow water conditions were reached. The wall wave travels at constant water height, and its profile follows closely the theoretical shape. Moreover, the linear relationship between the wave speed and wave amplitude was found to be very similar to the one predicted by the $\mathrm{KdV}$ theory. Although the initial swirl and the amount of the residual vorticity in the fluid affect the depth of water where the wave begins to appear, it does not seem to significantly influence the wave form and its celerity.
[1] J. Russell, Proc. R. Soc. Edinburgh 11, 319 (1844).

[2] K. Naugol'nykh and L. Ostrovsky, Nonlinear Wave Processes in Acoustics (Cambridge University Press, Cambridge, 1998).

[3] G. Lamb, Elements of Soliton Theory (Wiley, New York, 1980).
[4] E. Falcon, C. Laroche, and S. Fauve, Phys. Rev. Lett. 89, 204501 (2002).

[5] R. Grimshaw, Solitary Waves in Fluids (WIT Press, Southampton, Boston, 2007).

[6] W. K. Melville, G. G. Tomasson, and D. P. Renouard, J. Fluid Mech. 206, 1 (1989). 
[7] C. Katsis and T. Akylas, Phys. Fluids 30, 297 (1987).

[8] R. Grimshaw, Stud. Appl. Math. 73, 1 (1985).

[9] W. G. Pritchard, J. Fluid Mech. 42, 61 (1970).

[10] T. B. Benjamin, J. Fluid Mech. 28, 559 (1967).

[11] G. H. Vatistas, J. Fluid Mech. 217, 241 (1990).
[12] See Supplementary Material at http://link.aps.org/supplemental/ 10.1103/PhysRevE.84.037302 for movie showing a maturation of the free surface sloshing oscillation into azimuthal solitary surface wave during tank drainage. 